Sungwook Lee

Kwang-Jun Paik

http://dx.doi.org/10.21278/brod69107

ISSN 0007-215X

eISSN 1845-5859

\title{
URANS SIMULATION OF A PARTIALLY SUBMERGED PROPELLER OPERATING UNDER THE BOLLARD CONDITION
}

UDC 629.5.016: 629.5.035:519.6

Original scientific paper

\begin{abstract}
Summary
The performance of a partially submerged propeller under the bollard condition was assessed using numerical simulations based on URANS. The simulations were performed with varying propeller rotating speed and submergence depth. The propeller rotating speed was varied from 2 rps to $8 \mathrm{rps}$ with the interval of $2 \mathrm{rps}$ at the submergence depths of $h / R=$ $0.0,0.5$, and 1.0. Here, $h$ is the submergence depth from the free surface to the propeller shaft center and $R$ is the radius of the propeller. The thrust and torque losses were compared with the thrust and torque in the deep water condition. The thrust and torque decreased rapidly with increasing propeller rotating speed. The thrust and torque ratios were compared with the empirical formula showing generally good agreement. The hydrodynamic characteristics around the partially submerged propeller were investigated using numerical simulations.
\end{abstract}

Key words: $\quad$ Propeller; Free surface; Partially submerged propeller; Thrust loss; Air ventilation; Bollard condition

\section{Introduction}

Shipyards perform tests to check the operability of the main propulsion system and auxiliaries for a moored vessel in a quay before a sea trial. A propeller during the test would be partially submerged due to the limited water depth of the quay. An accurate estimation of the thrust and torque for the partially submerged propeller is needed to ensure the safety of mooring lines holding the ship during the test [1]. In addition, thrusters installed for dynamic positioning can work under heavy sea conditions. The vertical motions of a vessel or offshore structure and the waves bring the thrusters closer to the free surface, making them more susceptible to ventilation [2]. A surface-piecing propeller is one of the most efficient propulsion systems for high-speed vessels. They can use a larger propeller size because it is not limited by the minimum blade tip clearance from the hull or the maximum vessel draft. Moreover, they can avoid cavitation damage because the propeller operates under ventilated conditions by drawing air from the free surface [3]. 
The common characteristics of the above three propellers are working with air ventilation under partially submerged condition. On the other hand, the propeller working in the quay and the thruster for dynamic positioning are different from the surface-piercing propeller in the inflow velocity of a fluid because they work in a fixed-position. The propeller operation with a zero advance speed is called the bollard condition. Thrust and torque are very large under the bollard condition. Large torque and sudden variations of the load conditions can be caused by intermittent ventilations, which occur on the partially submerged propeller experiencing continuous cycles of water-exit and re-entry [4].

The partially submerged propeller experiences thrust loss due to the air ventilation phenomenon. The thrust loss can be caused by the loss of propeller disk area, Wagner effect, thrust loss due to wave-making by the propeller, and air ventilation [5]. Kempf [6] first studied the air ventilation effect on the propellers. Since then, there have been many studies of the air ventilation of propellers [7 12]. Koushan [13] focused on the thrust loss due to air ventilation as well as the effects of air ventilation on the dynamics of the blade thrust and torque about the propeller shaft. The experiment was performed with a ducted propeller and an open propeller with a heave motion. Koushan et al. [2] examined the effects of waves and the propeller loading of an open pushing thruster though an experiment. They showed that the effects of the wave height are significant, particularly for the sub critical region (advance coefficients larger than 0.4), where higher waves lead to larger thrust loss. Califano \& Steen [14] proposed two main ventilation mechanisms depending on the propeller submergence, loading, and advance coefficient. One is the free-surface vortex at the deeper submergence, and the other is the tip vortex at moderate submergence. These two mechanisms can exist separately or simultaneously. Park et al. [1] took the model experiment with two partially submerged propellers to derive the empirical formula to predict the thrust and torque losses according the submergence depth and ventilation number.

Numerical methods based on potential theory were developed to simulate the air cavity sucked down from the free surface at the early stages $[3,12]$. On the other hand, with the development of computer systems, research using URANS (Unsteady Reynolds averaged Navier-Stokes) simulations is becoming increasingly popular. Califano \& Steen [4] simulated a fully submerged propeller $(h / R=1.4)$ working at a high loading $\left(J_{A}=0.1\right)$ using a URANS code. Kozlowska et al. [15] compared the URANS simulation results with the experimental data performed using a range of advance coefficients $\left(J_{A}=0.0 \sim 0.6\right)$ under the fully submerged condition $(h / R=1.5)$. On the other hand, Park et al. [1] showed the possibility of URANS simulation for a partial submerged propeller working under the bollard condition.

In this study, numerical simulations using URANS were carried out to investigate the flow characteristics with air ventilation according to the submergence depth and propeller rotating speed. The propeller rotating speed and submergence depth $(h / R)$ were respectively 2 rps to 8 rps at 2 rps intervals and 0.0, 0.5, and 1.0. Here, $h$ is the submergence depth from the free surface to the propeller shaft center and $R$ is the radius of the propeller. The time series of the thrust and torque of one blade during a single revolution were investigated to understand the effects of air ventilation.

\section{Mathematical and numerical models}

\subsection{Governing equations}

The governing equations for the numerical simulation are the continuity equation and the incompressible URANS equations. The integral forms of the equations are as follows: 
$\frac{d}{d t} \int_{\Omega} \rho d \Omega+\int_{S} \rho u_{i} n_{i} d S=0$

$\frac{d}{d t} \int_{\Omega} \rho u_{i} d \Omega+\int_{S} \rho u_{i} u_{j} n_{j} d S=\int_{S}\left(\tau_{i j} n_{j}-p n_{i}\right) d S+\int_{\Omega} \rho b_{i} d \Omega$

where $\rho$ and $p$ are density and pressure, respectively; $u_{i}$ is the velocity tensor and $b_{i}$ is the tensor of body forces; and $\tau_{i j}$ is the effective stress of the viscosity and turbulence, defined as $\tau_{i j}=\mu_{e}\left[\left(\frac{\partial u_{i}}{\partial x_{j}}+\frac{\partial u_{j}}{\partial x_{i}}\right)-\frac{2}{3} \delta_{i j} \frac{\partial u_{k}}{\partial x_{k}}\right]$

\subsection{Numerical methods}

Commercial CFD software STAR-CCM+ 11.04 was used for the simulations in this study. The STAR-CCM+ is based on the finite volume method. The convection and diffusion terms were discretized using a $2^{\text {nd }}$ order upwind scheme and a central difference scheme, respectively. For pressure-velocity coupling, the SIMPLE (Semi-Implicit Method for PressureLined Equation) algorithm was implemented. The SST (Shear Stress Transport) $k-\omega$ model was applied to a turbulence model. The VOF (Volume of Fluid) model based on HRIC (High Resolution Interface Capturing) scheme was implemented to capture the behavior of the free surface due to propeller operation.

\section{Numerical simulation set-up}

\subsection{Model propeller}

The model propeller used in this study is KP505, which was designed by the Korea Research Institute of Ships and Ocean Engineering (KRISO) for the KRISO container ship called KCS. The diameter of the full-scale propeller was $7.9 \mathrm{~m}$ and the number of blades was 5. The diameter of the model propeller was $250.0 \mathrm{~mm}$ from a scale ratio of 31.6. Table 1 and Figure 1 present the principal particulars and drawing of the model propeller, respectively.

Table 1 Principal particulars of the model propeller (KP505)

\begin{tabular}{|l|l|}
\hline Diameter $(\mathrm{mm})$ & 250.0 \\
\hline Scale ratio & 31.6 \\
\hline No. of blades & 5 \\
\hline P/D (mean) & 0.95 \\
\hline Ae/Ao & 0.800 \\
\hline Hub ratio & 0.180 \\
\hline Section & NACA66 \\
\hline
\end{tabular}

\subsection{Grid system}

Figure 2 shows the computational domain and grid system for the numerical simulation. The trimmer mesh scheme using unstructured grids was applied to generate the grids around the model propeller. The grid system consists of a propeller block and background block. The propeller block surrounding the propeller blade and hub is a sliding mesh and rotates along a sliding mesh interface to consider the relative rotating motion of the propeller to the free surface. In addition, the boundary layer on the propeller blade surface was constructed using a prism layer so that the dimensionless wall distance was less than $1\left(y^{+}<1\right)$. 


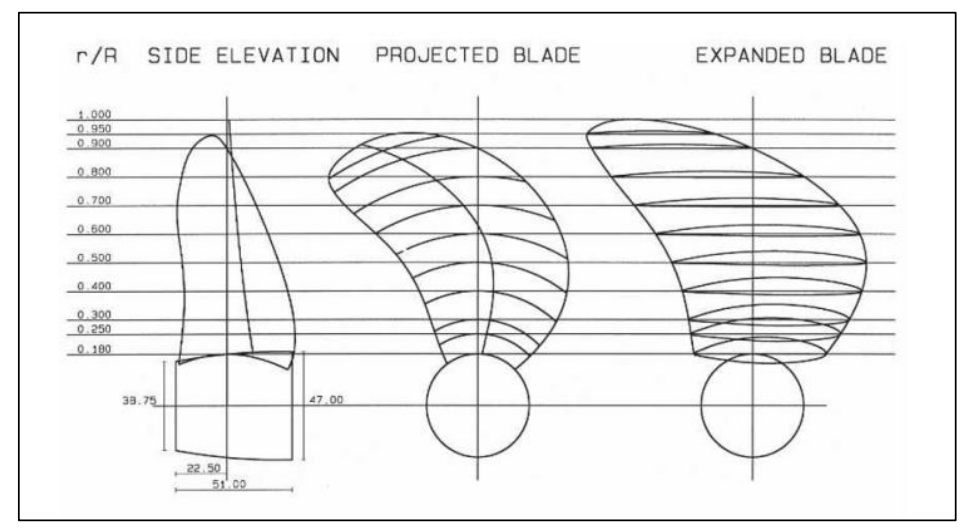

Fig. 1 Propeller drawing of the model propeller (KP505)

The grids around the tip and root of the model propeller have an additional layer to capture the tip and root vortices. Refinement grids were applied to capture the behavior of the free surface. The number of grids for the propeller block and background are $1.58 \mathrm{M}$ and $5.30 \mathrm{M}$, respectively. On the other hand, the reinforced grids for the free surface were eliminated for the propeller open water (POW) simulations in the deep water. The number of background grid without the refinement grid for the free surface are $4.2 \mathrm{M}$. The ratio of the submergence depth of the model propeller $(h / R)$ is defined as the ratio between the depth $(h)$ from the free surface to the propeller shaft and the radius of the model propeller $(R)$, which are depicted in Fig. 2.
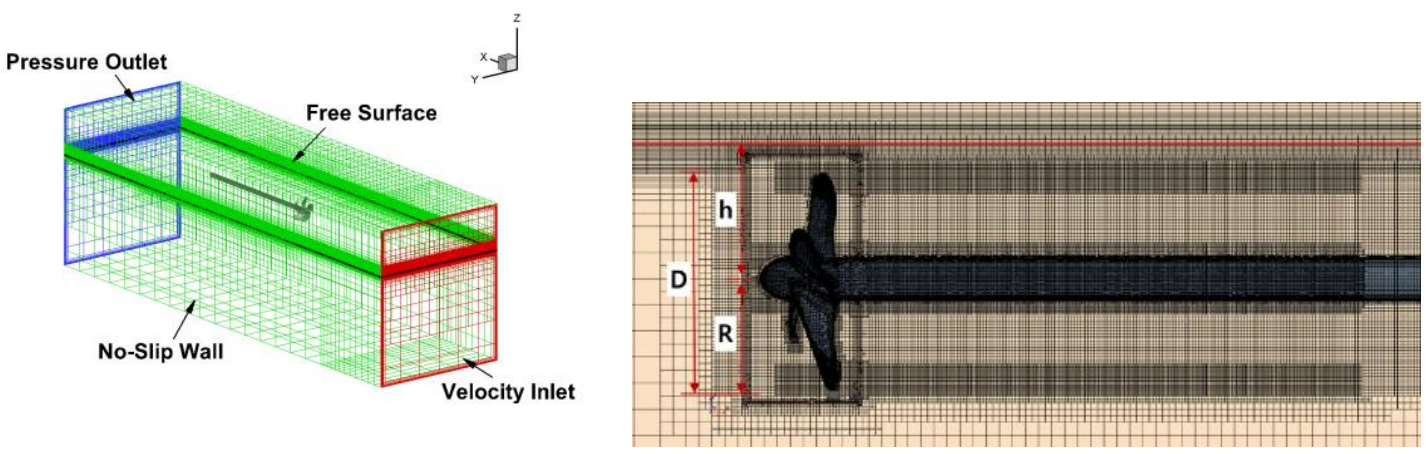

Fig. 2 Computational domain and grid system

The propeller blade angle begins from the top position toward the propeller rotation direction, as illustrated in Figure 3. When a key blade is located at the top, the propeller blade angle is defined as $0^{\circ}$.

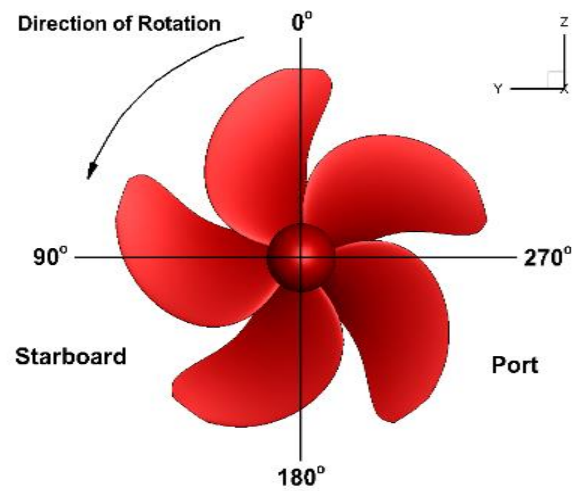

Fig. 3 Definition of the propeller blade angle 


\section{Results and discussion}

\subsection{Validation of numerical simulation}

The numerical simulation method applied in the study was validated by a comparison with the propeller open water (POW) characteristics measured in a model experiment. The experiment was performed in KRISO using a model propeller. The diameter of the model propeller and the rotating speed was $D=250.0 \mathrm{~mm}$ and $n=14 \mathrm{rps}$, respectively. The speed of carriage towing the POW test equipment was varied according to the advance coefficient $\left(J_{A}=V_{A} / n D\right)$. The numerical simulations were performed under the same conditions as the model test.

The thrust $\left(K_{T}\right)$ and torque $\left(K_{Q}\right)$ coefficients and open water efficiency $\left(\eta_{O}\right)$ using the SST $k-\omega$ model and $k-\varepsilon$ model for the turbulence model are compared in Figure 4 . The thrust and torque coefficients generally show good agreement with the experiment, even though the slope of the thrust and torque coefficients are slightly different from the experiment. Nevertheless, they showed very good agreement around the low advance coefficients. Because there was no significant difference between two turbulence models, the SST $k-\omega$ model was applied to the simulations for the partially submerged propeller. Table 2 shows the errors between the CFD simulation using the SST $k-\omega$ model and experiment.

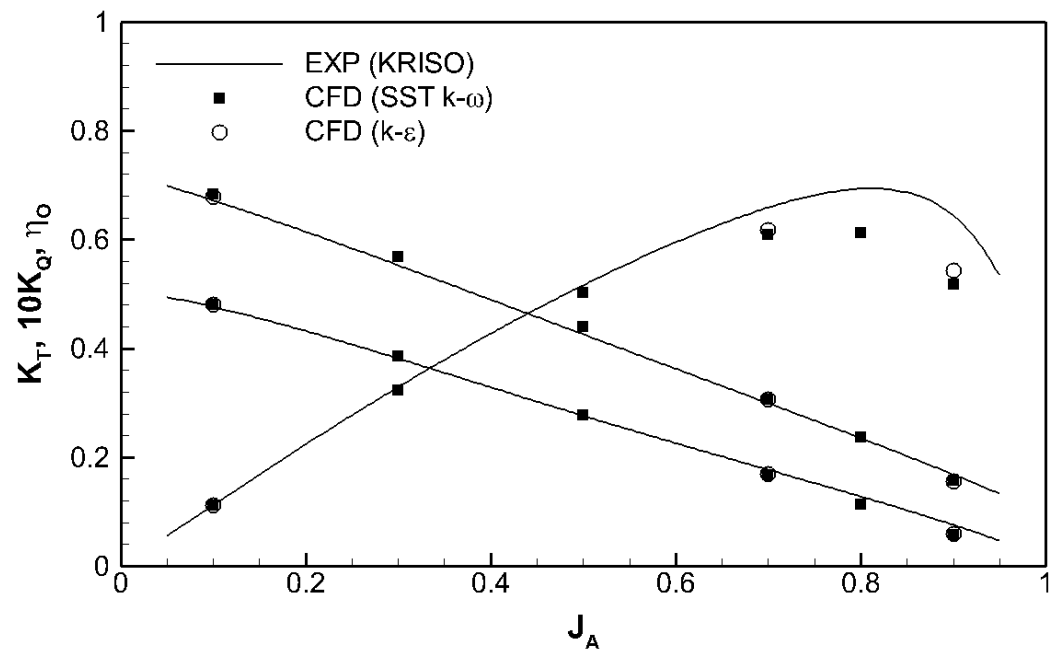

Fig.4 Comparison of the propeller open water characteristics in the deep water between CFD and experiment

Table 2 Comparison of the thrust and torque coefficients in the deep water between CFD and experiment

\begin{tabular}{|c|c|c|c|c|c|c|}
\hline \multirow{2}{*}{$J_{A}$} & \multicolumn{2}{|c|}{ CFD $(\mathrm{SST} k-\omega)(\mathrm{a})$} & \multicolumn{2}{c|}{ KRISO (b) } & \multicolumn{2}{c|}{ Error\% (a/b-1) } \\
\cline { 2 - 7 } & $K_{T}$ & $10 K_{Q}$ & $K_{T}$ & $10 K_{Q}$ & $K_{T}$ & $10 K_{Q}$ \\
\hline 0.1 & 0.480 & 0.684 & 0.476 & 0.672 & $0.8 \%$ & $1.7 \%$ \\
\hline 0.3 & 0.385 & 0.569 & 0.381 & 0.553 & $1.1 \%$ & $3.0 \%$ \\
\hline 0.5 & 0.278 & 0.440 & 0.276 & 0.426 & $0.4 \%$ & $3.4 \%$ \\
\hline 0.7 & 0.168 & 0.307 & 0.177 & 0.299 & $-5.1 \%$ & $2.7 \%$ \\
\hline 0.8 & 0.114 & 0.237 & 0.128 & 0.235 & $-11.1 \%$ & $0.7 \%$ \\
\hline 0.9 & 0.057 & 0.158 & 0.076 & 0.168 & $-24.8 \%$ & $-6.4 \%$ \\
\hline
\end{tabular}




\subsection{Simulation conditions}

In the model test, the kinematic similarity is satisfied from the same advance coefficient using a full-scale propeller. To satisfy the dynamic similarity, the Froude number, Reynolds number, Weber number, and ventilation number should be identified.

The ventilation number is usually defined using the relationship between pressure and inertia, such as the cavitation number.

$\sigma_{v}=\frac{2 g h_{t i p}}{V_{R}^{2}}$

In the model test for the bollard condition, the inflow velocity is zero, which means the advance coefficient is zero $\left(J_{A}=0\right)$. Therefore, the ventilation number is expressed below under the bollard condition due to the zero inflow velocity.

$\sigma_{v}=\frac{2 g h_{t i p}}{(\pi n D)^{2}}$

where $h_{\text {tip }}$ is defined as $h+R$ to compare with previous research (Park et al. [1]). The ventilation number decreases with increasing propeller rotating speed and decreasing submergence depth. The similarity for the ventilation number is satisfied automatically when the similarities for the geometry and Froude number are satisfied.

Park et al. [1] performed model tests with 5-blade and 6-blade propellers at five propeller rotating speeds $(2,4,6,8,10 \mathrm{rps})$ and seven submergence depths $(h / R=0.0,0.5,1.0,1.5,2.0$, 2.5, and 3.0). The numerical simulations in this study were performed by varying the propeller rotating speed $(2,4,6,8 \mathrm{rps})$ at three submergence depths $(h / R=0.0,0.5$, and 1.0). The maximum propeller rotating speed was determined from the Froude similarity due to the interaction with the free surface, while the propeller rotating speed in the deep water is usually determined to perform the model test in a high Reynolds number. As a result, the propeller rotating speed of $8 \mathrm{rps}$ in the model scale corresponds to $85.4 \mathrm{rpm}$ in the full scale. It is quite reasonable rotating speed when the maximum propeller rotating speed of large commercial vessels is around $100 \mathrm{rpm}$. Table 3 lists the ventilation number for each simulation condition. While the model tests in Park et al. [1] were carried out under the bollard condition of zero inflow velocity $\left(J_{A}=0\right)$, the numerical simulations were conducted with a very slow inflow velocity $\left(J_{A}=0.01\right)$ to improve the numerical stability.

Table 3 Ventilation number of the simulation conditions

\begin{tabular}{|c|c|c|c|}
\hline${ }_{r p s} h / R$ & 0.0 & 0.5 & 1.0 \\
\hline 2 & 0.994 & 1.491 & 1.988 \\
\hline 4 & 0.248 & 0.373 & 0.497 \\
\hline 6 & 0.110 & 0.166 & 0.221 \\
\hline 8 & 0.062 & 0.093 & 0.124 \\
\hline
\end{tabular}

The Weber number for the effect of surface tension is defined as

$W_{e}=n D \sqrt{\frac{\rho D}{s}}$

where $s$ is the surface tension of water. Shiba (1953) suggested a criterion $\left(W_{e}>180\right)$ to neglect the influence of surface tension. On the other hand, the Weber number at $8 \mathrm{rps}$ is approximately 118, which is smaller than the criterion. The effect of the surface tension on the simulation result was investigated, as shown in Figure 5. The convergence of simulation with 
the surface tension was very poor as compared to the simulation without the surface tension, even though very smaller relaxation factors was applied to the simulation with the surface tension. Therefore, the effect of surface tension was not implemented in the other simulations.

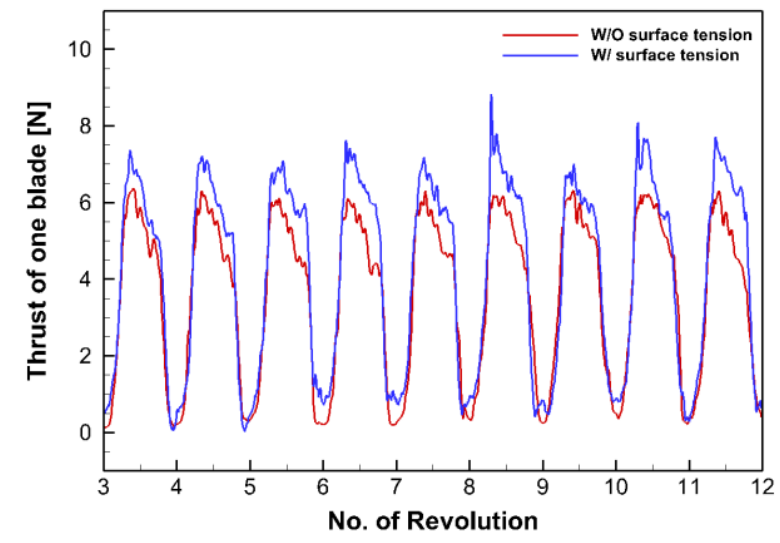

Fig. 5 Comparison of the thrust variations without and with surface tension

Before the simulations for all cases, the size of time step was investigated with the time steps of 1 degree and 2 degrees for the case of 4 rps and $h / R=0.5$, as shown in Figure 6 . They shows very good agreement even though the variations of thrust and torque of 1 degree are a little more fluctuating. Therefore, the simulations for the other conditions were performed with the time step corresponding to 2 degrees because it is enough to investigate the tendency of thrust and torque losses due to the air ventilation. When the variation of thrust ratio during nine revolutions after the very first three revolutions are compared at $4 \mathrm{rps}$ and $h / R=0.5$, as shown in Figure 7, the deviation is not significant. The thrust ratio was based on the thrust under the deep water condition in the same propeller rotating speed. Therefore, the variations of thrust and torque during a single revolution in the same time period are compared for the other conditions.

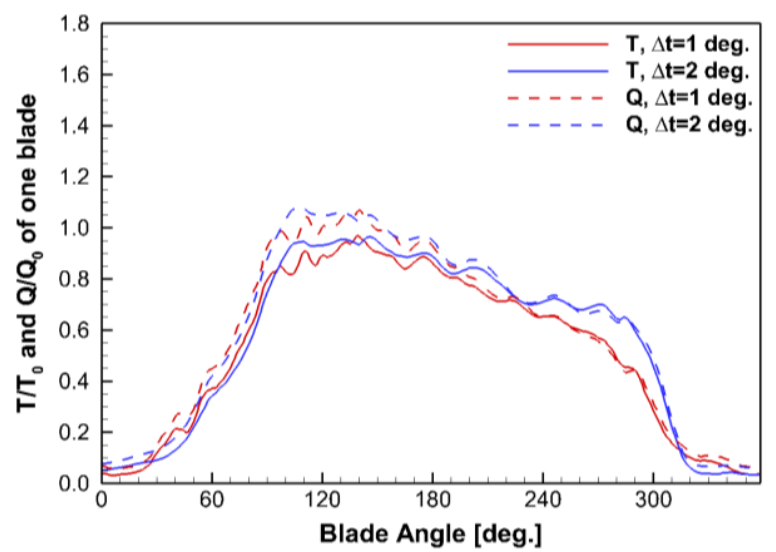

Fig. 6 Comparison of the thrust and torque ratios according to the time step

A sharpening factor $(\kappa)$ can be adjusted in STAR-CCM+, which is the factor to reduce the level of diffusion in the simulation. When the sharpening factor is 0 , there is no reduction in numerical diffusion. When the sharpening factor is 1 , on the other hand, there is no numerical diffusion with a very sharp interface between the two phases (STAR-CCM+ User Guide). To determine the optimal sharpening factor, three sharpening factors were tested with 4 rps at $h / R=0.5$. Figure 8 shows the iso-surfaces of the free surface according to the number of sharpening factor. The blue iso-surface indicates the surface where the volume fraction of water is 0.5 . The interface between water and air at the sharpening factor of 0.0 is relatively smoother than the other higher sharpening factors. When the variations of the thrust and torque of one 
blade during a single revolution are compared, as shown in Figure 7, the fluctuations of the thrust and torque increase in the higher sharpening factors. Therefore, a sharpening factor of 0.5 was selected to compromise the shape of interface and the variations of thrust and torque, and then it was applied to all simulations in this study.

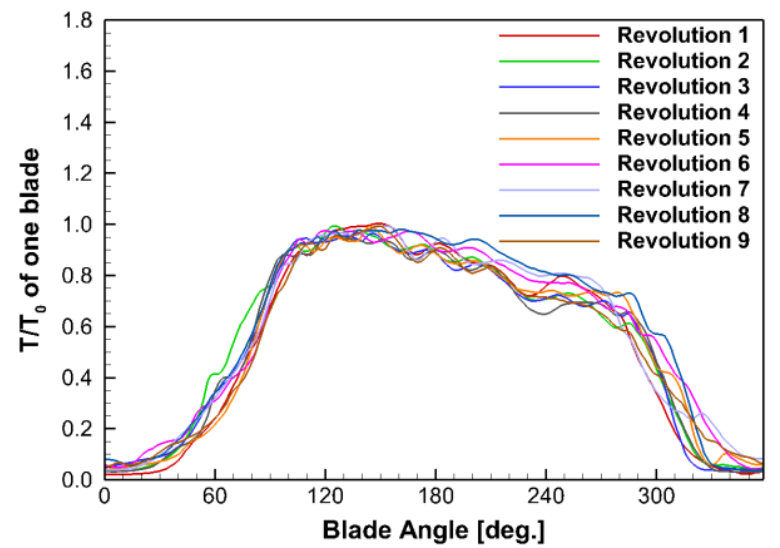

Fig. 7 Variation of the thrust ratio according to the revolution

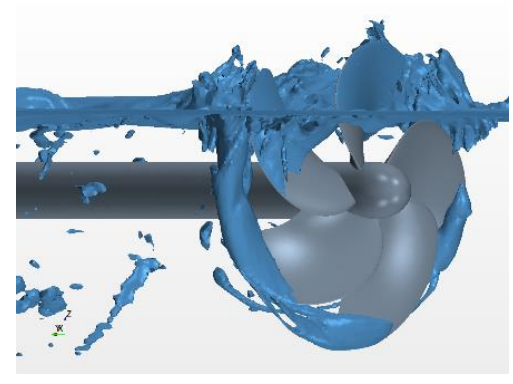

(a) $\kappa=0.0$

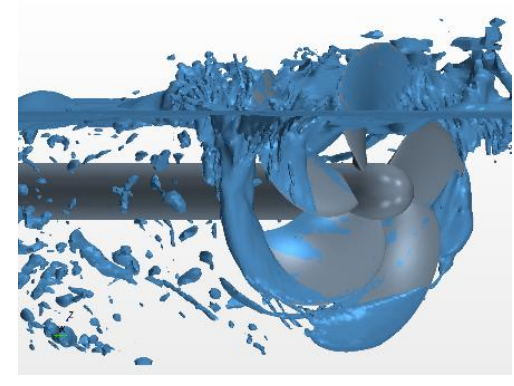

(a) $\kappa=0.5$

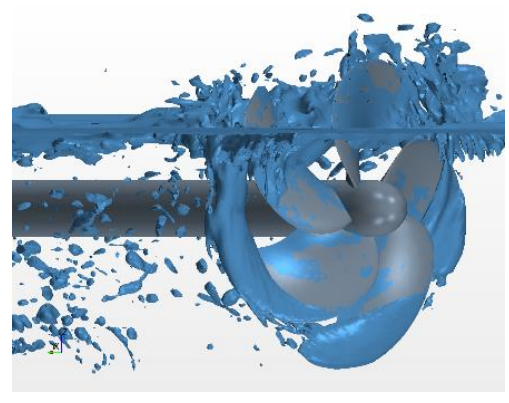

(a) $\kappa=0.9$

Fig. 8 Oblique view of the iso-surface of the free surface according to the sharpening factor $(\kappa)$
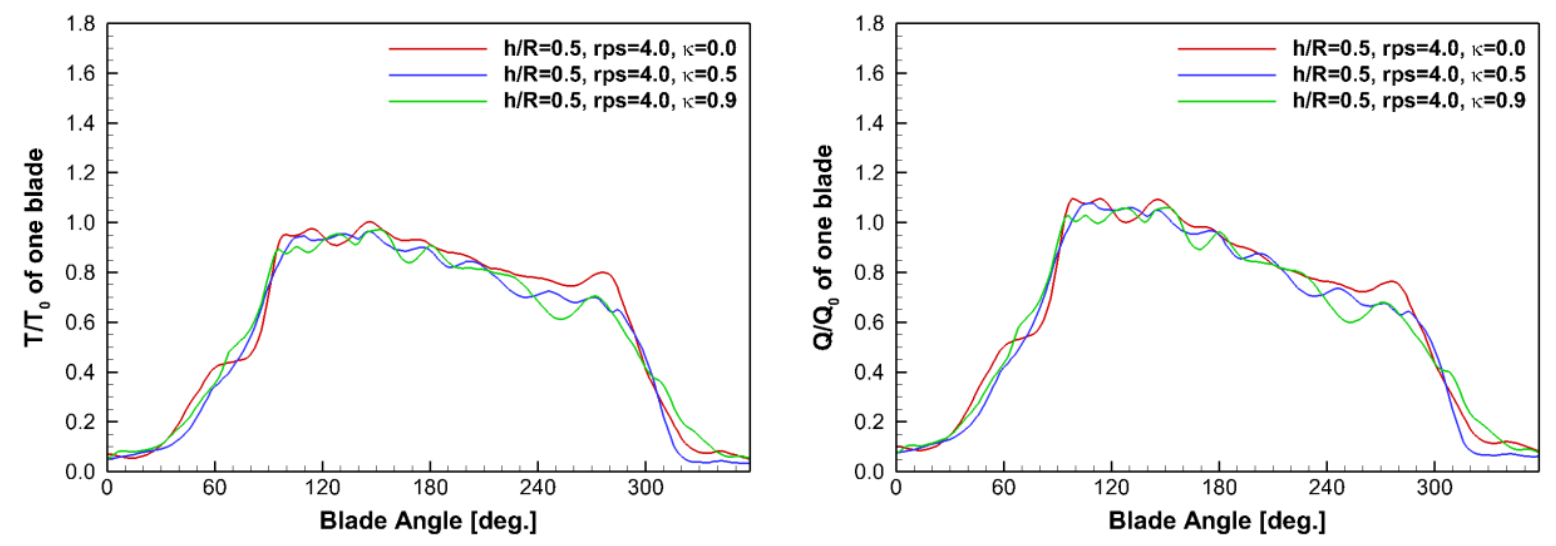

Fig. 9 Comparison of the thrust and torque ratios according to the sharpening factor

\subsection{Simulation results}

Figure 10 compares the instantaneous iso-surfaces of the free surface under each simulation condition. The iso-surface under the free surface is the air cavity sucked down to the water, and the iso-surface above the free surface is the water droplet splashed into air. The angle of the blade located on the top positon is zero. The next blade is located at $72^{\circ}$ because the number of blades of this propeller is 5 . 
2 rps
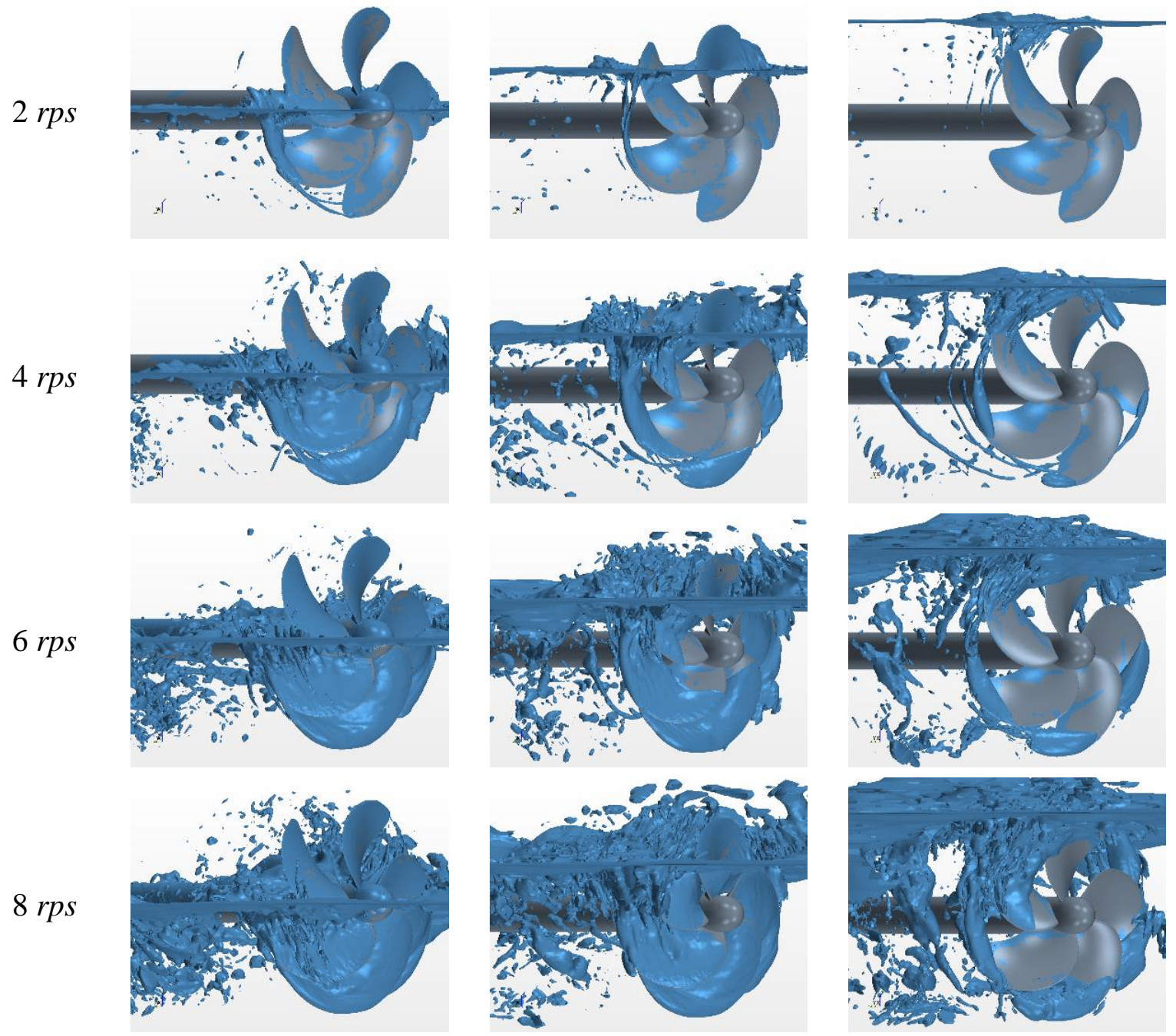

(a) $h / R=0.0$

(b) $h / R=0.5$

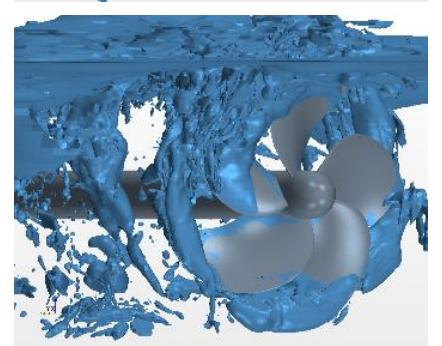

(c) $h / R=1.0$

Fig. 10 Oblique view of the iso-surface of the free surface according to the submergence depth

When the propeller rotating speed is 2 rps, the air bubbles sucked down to the water follow the tip vortex of the blade regardless of the submergence depth. On the other hand, because the relative position of the blade to the free surface and the hydrostatic pressure change according to the submergence depth, the amount of air bubbles ventilated due to the tip vortex decreases with increasing submergence depth. In particular, when $h / R$ is 1.0 , the tip vortex is not observed clearly because there is no water-exit and re-entry of the blade.

At the same submergence depth, air ventilation and wave-making increase with increasing propeller rotating speed. The amount of water droplets splashed by the blade exit and re-entry increase at a higher propeller rotating speed. The water droplet was observed more in $h / R=0.5$ than $h / R=0.0$ at a higher propeller rotating speed. On the other hand, the amount of the water droplet decreases in $h / R=1.0$ because the blades do not pierce the free surface directly. Finally, the air cavity covers the entire blades under the free surface in 6 rps at $h / R=0.0$ and $8 \mathrm{rps}$ at $h / R=0.5$, while the air cavity does not cover the entire blades at any propeller rotating speed at $h / R=1.0$.

Figure 11 compares the thrust and torque ratios according to the submergence depth with those under the deep water condition in the same propeller rotating speed. The curves tend to be oscillating particularly at higher propeller rotating speeds because the curves show just one 
revolution event, even though the behavior of the air cavity varies at each revolution due to the instability of the air ventilation, as shown in Figure 7.

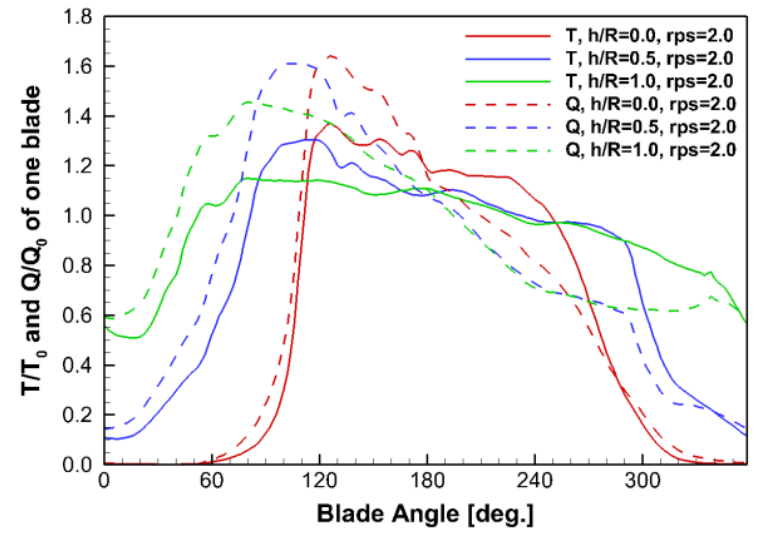

(a) $2.0 \mathrm{rps}$

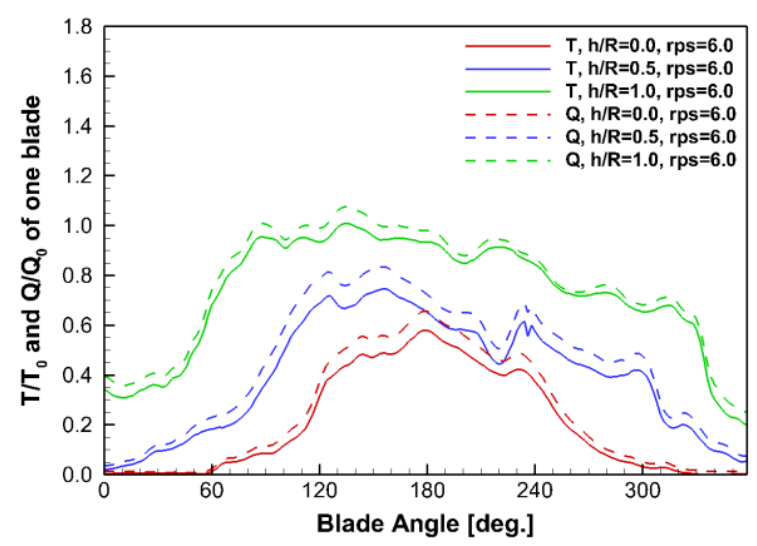

(c) $6.0 \mathrm{rps}$

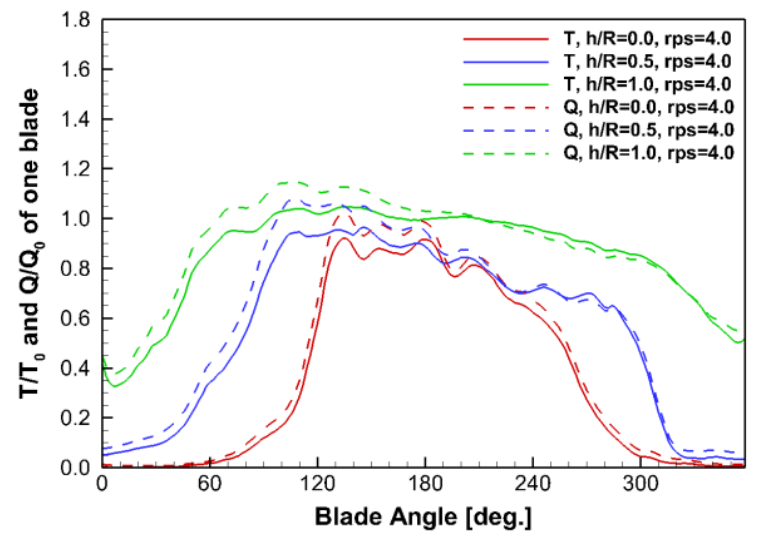

(b) $4.0 \mathrm{rps}$

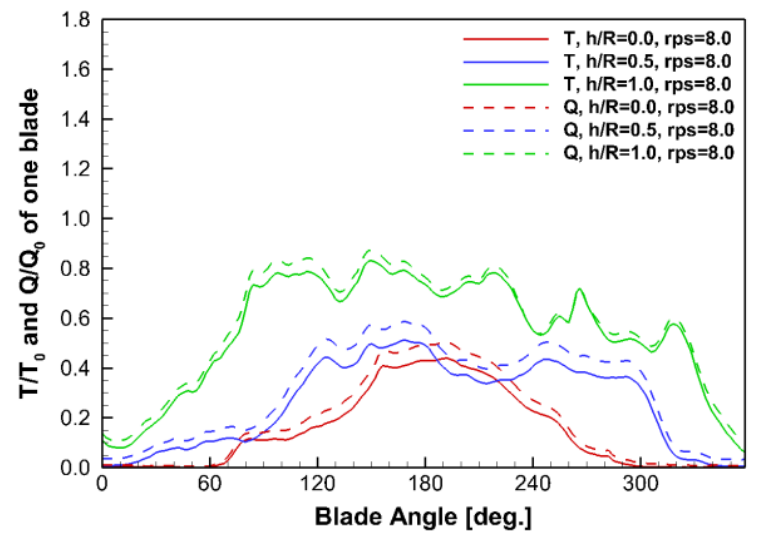

(d) $8.0 \mathrm{rps}$

Fig. 11 Variation of the thrust and torque ratios according to the submergence depth

At $h / R=0.0$ and $2 \mathrm{rps}$, the thrust was zero from $330^{\circ}$ to $60^{\circ}$, which is the range where the blade is out of the water, and the thrust and torque increase rapidly due to the blade re-entry. The maximum ratios of thrust and torque are greater than one, which means that the thrust and torque is bigger than those under the deep water condition. This phenomenon was observed in the experiments conducted by Califano and Steen [14] and Kozlowska et al. [15]. With increasing submergence depth, the range of thrust recovery extends and the slope of the thrust increment at the blade entry region becomes gentle. The thrust loss is larger than the torque loss at the blade entry region, while the torque loss is larger than the thrust loss at the blade exit region at all submergence depths. On the other hand, the thrust loss is generally larger than the torque loss and the difference between thrust and torque losses is not significant at the other propeller rotating speeds except for 2 rps.

The maximum thrust and torque ratios were approximately one at all submergence depths at 4 rps. Air cavity sucked down the free surface covers the entire blade areas under the free surface at $h / R=0.0$ in $4 \mathrm{rps}$, as shown in Figure 10. As a result, the maximum thrust ratio at those conditions was less than one. On the other hand, the maximum thrust and torque ratios of $h / R=0.0$ and $h / R=0.5$ at 6 rps were smaller than that due to ventilation. In addition, the maximum values at $h / R=1.0$ were less than one at 8 rps. As shown in Figure 10, the air bubbles ventilated at $h / R=1.0$ at 8 rps covered outer radii region of the blade positioned at the bottom. 
The thrust and torque ratios shown in Figure 11 are averaged during a single revolution and are summarized in Table 4. The thrust and torque ratios at $h / R=0.0$ were larger than the propeller disk area under the free surface at $2 \mathrm{rps}$, and they reached less than $20 \%$ at $8 \mathrm{rps}$. Furthermore, the thrust ratio at $h / R=1.0$ was smaller than $60 \%$ at $8 \mathrm{rps}$. As explained in Figure 11, the thrust loss was larger than the torque loss in all cases, which means a decrease in propeller efficiency. The propeller efficiency decreased with increasing propeller rotating speed and decreasing submergence depth.

Table 4 Thrust and torque losses according to the submergence depth and propeller rotating speed

\begin{tabular}{|c|c|c|c|c|}
\hline$h / R$ & $\mathrm{rps}$ & $T / T_{0}$ & $Q / Q_{0}$ & $\eta / \eta_{0}$ \\
\hline \multirow{4}{*}{0.0} & 2 & 0.578 & 0.588 & 0.982 \\
\cline { 2 - 5 } & 4 & 0.360 & 0.400 & 0.899 \\
\cline { 2 - 5 } & 6 & 0.202 & 0.239 & 0.847 \\
\hline \multirow{4}{*}{0.5} & 8 & 0.146 & 0.176 & 0.827 \\
\cline { 2 - 5 } & 2 & 0.789 & 0.801 & 0.984 \\
\cline { 2 - 5 } & 4 & 0.549 & 0.599 & 0.916 \\
\cline { 2 - 5 } & 6 & 0.407 & 0.468 & 0.869 \\
\hline \multirow{5}{*}{1.0} & 2 & 0.259 & 0.314 & 0.824 \\
\cline { 2 - 5 } & 4 & 0.944 & 0.950 & 0.993 \\
\cline { 2 - 5 } & 6 & 0.822 & 0.849 & 0.968 \\
\cline { 2 - 5 } & 8 & 0.710 & 0.751 & 0.945 \\
\hline
\end{tabular}

Figure 12 compares the pressure distributions of the blades at $h / R=0.0$ and $2 \mathrm{rps}$ with those under the deep water condition. The area of negative pressure on the suction side was larger due to the air cavity on the blade surface after the blade entered the water. The areas of the positive pressure on the pressure were also larger than those under the deep water condition for the same reason. This can explain why the maximum thrust and torque ratios at $h / R=0.0$ and 2 rps can be greater than one, as shown in Figure 11. At $h / R=0.5$ and $h / R=1.0$, the areas of the pressure and suction side on the fully submerged blade were also larger than those under the deep water condition. In addition, at $h / R=1.0$, the pressure of the blade at the top was affected by ventilation, even though it did not penetrate the free surface.

Figure 13 shows the variation of the thrust and torque ratios according to the propeller rotating speed at each submergence depth to compare the effect of the propeller rotating speed more clearly. The distribution of the thrust and torque ratios showed a similar tendency at the same submergence depth; even the magnitude differs according to the propeller rotating speed. The change in thrust ratio according to the propeller rotating speed depends on the submergence depth. The reduction rate of the thrust ratio decreases with increasing propeller rotating speed at $h / R=0.0$, whereas the reduction rate of the thrust ratio is almost constant with the variation of propeller rotating speed at $h / R=0.5$. The reduction rate of the thrust ratio at $2 \sim 6 r p s$ of $h / R=1.0$ is not as distinct as that at the other submergence depths.

The thrust and torque ratios summarized in Table 4 were plotted with the regression curves of Park et al. [1]. The regression was performed based on the data measured from model 
tests with 5-blade and 6-blade propellers. The regression formula considers the effects of the ventilation number and submergence depth.

$\beta_{O}=1-\frac{\cos ^{-1}\left(\frac{h}{R}\right)}{\pi}+\frac{h}{\pi R} \sqrt{1-\left(\frac{h}{R}\right)^{2}}, \quad\left(0.0 \leq \frac{h}{R} \leq 1.0\right)$
$\beta_{Q}=\beta_{O}\left[1-\frac{1}{28.6\left(1-\frac{0.18 h / R}{0.18+h / R}\right) \sigma_{v}+1}\right]$

Deep water
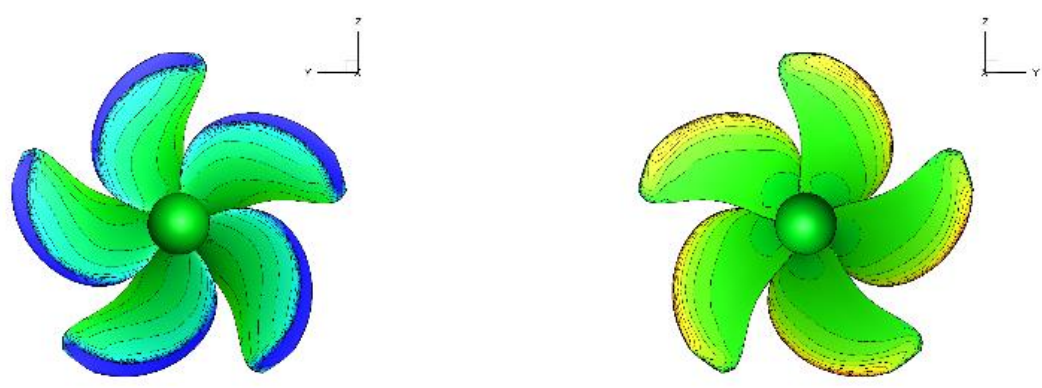

$h / R=0.0$
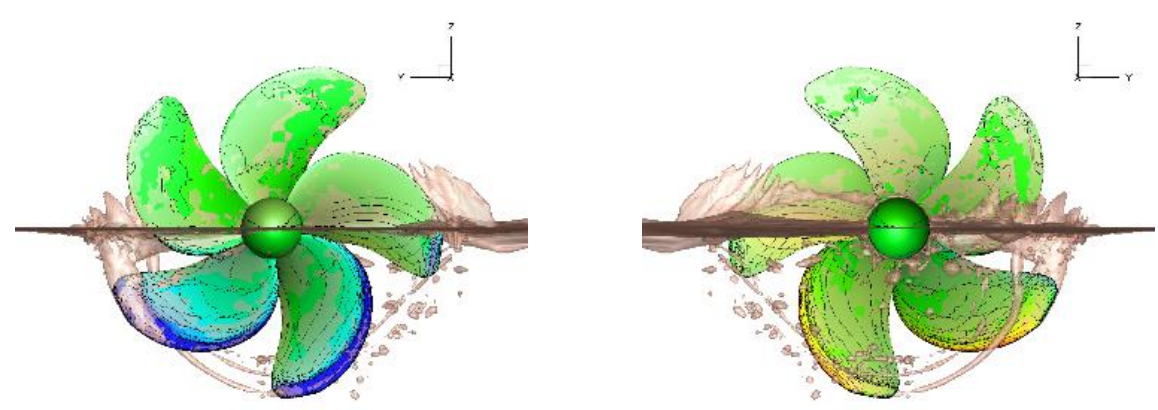

$h / R=0.5$
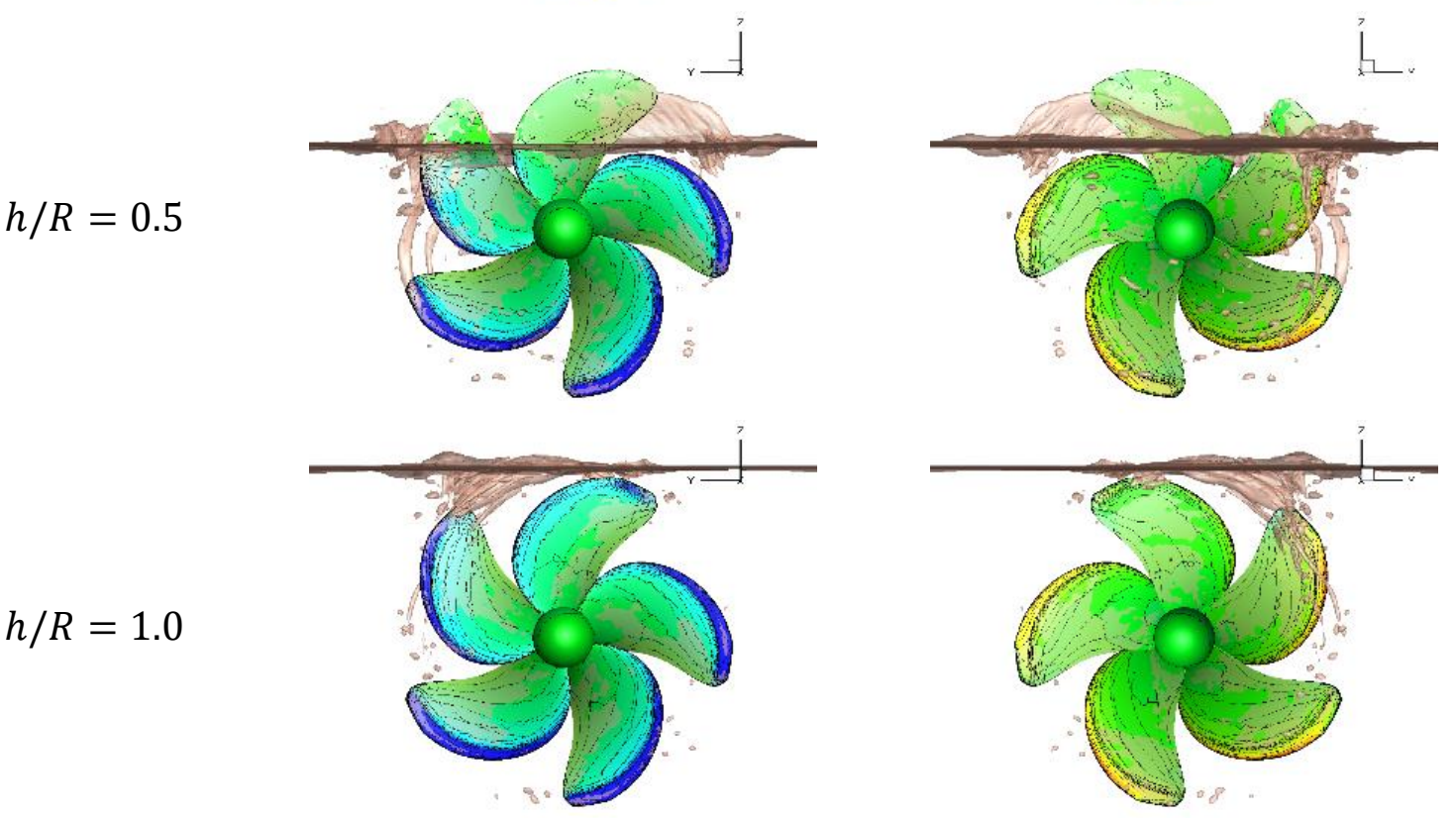

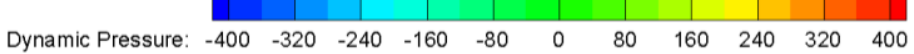

Fig. 12 Pressure contours of back (left) and face (right) sides according the propeller rotating speed at $2.0 \mathrm{rps}$ 


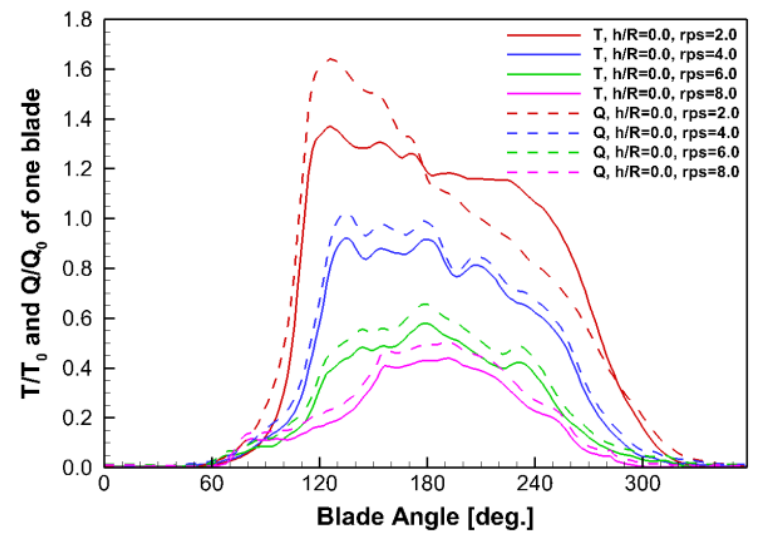

(a) $h / R=0.0$

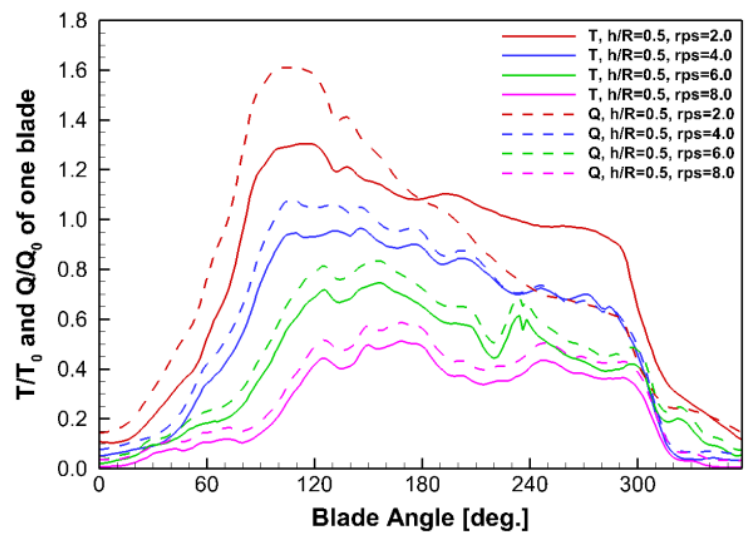

(b) $h / R=0.5$

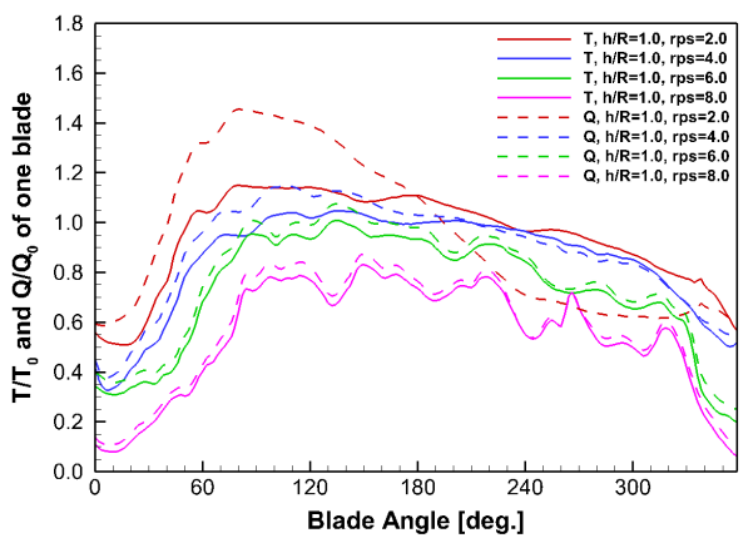

(c) $h / R=1.0$

Fig. 13 Variation of the thrust and torque ratios according the propeller rotating speed

The effects of the wetted-disk area $\left(\beta_{O}\right)$ is determined by the submergence depth, which was suggested by Fleischer [9]. $\beta_{Q}$ is the torque ratio, which includes the effects of wave making and air ventilation according to the submergence depth as well as the effect of the loss of propeller disc area. The effects of wave making and air ventilation tend to decrease due to the lower propeller loading in a smaller submergence depth. The thrust ratio is defined as $\beta_{T}=$ $\beta_{Q}{ }^{1 / m}$ using the torque ratio. Here, $m$ is a constant for the relationship between the thrust ratio and torque ratio. Minsaas [11] suggested a value between 0.8 and 0.85 for the constant, $m$, whereas Park et al. [1] obtained 0.9 for $m$ from their experiment. The constant, $m$, from the thrust and torque ratios in Table 4 is between 0.83 to 0.97 . As a result, the average value is approximately 0.9 , and it coincides with the value obtained from Park et al. [1].

Figure 14 compares the thrust and torque ratios obtained from the numerical simulation with the experiment data and regression curves reported by Park et al. [1]. The experimental data show an approximately $10 \%$ difference between the 5-blade and 6-blade propellers. The thrust and torque ratios of the 5-blade propeller is bigger than those of the 6-blade propeller because the loading of the 5-blade propeller is smaller than that of the 6-blade propeller. The results from the numerical simulation generally show a good tendency with the regression curves. The thrust and torque ratios at $h / R=1.0$ show very good agreement with the experimental results of a 5-blade propeller even at a lower ventilation number. On the other hand, under the partially submerged condition, the numerical simulation tends to under-predict at lower ventilation numbers and over-predict at higher ventilation numbers. The numerical 
simulation results of $h / R=0.0$ and $h / R=0.5$ were closer to the experimental results of the 6-blade propeller rather than the 5-blade propeller at the lower ventilation number.
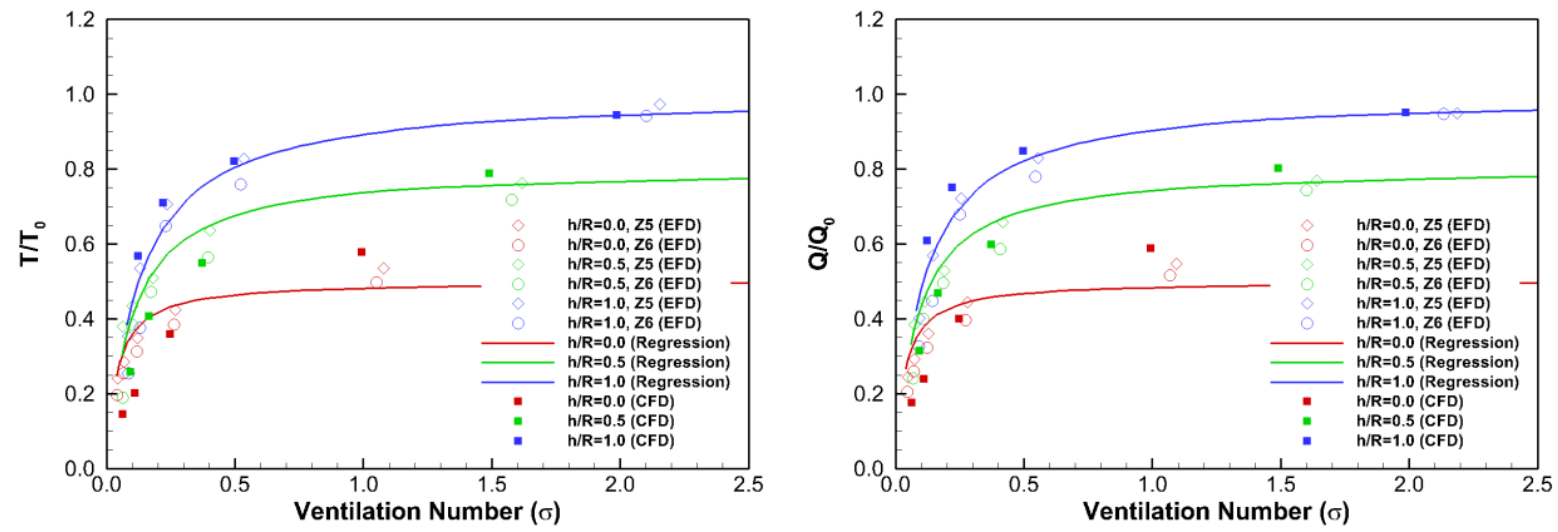

Fig. 14 Comparison of the thrust and torque losses according to the ventilation number and submergence depth (The propeller rotating speeds in EFD and CFD are $n=2,4,6,8,10$ rps)

\section{Conclusions}

The effects of air ventilation on the propeller performance according to the variations of submergence depth and propeller rotating speed in bollard condition were investigated using URANS simulations. The propeller rotating speed was varied from 2 rps to 8 rps with intervals of 2 rps at submergence depths of $h / R=0.0,0.5$, and 1.0.

Initially, the numerical method applied to this study was validated by a comparison with the experimental data of a POW test performed at KRISO. The thrust and torque coefficients generally showed good agreement with the experimental data, particularly the low advance coefficients.

The numerical simulation with the partially submerged propeller shows realistically the physical phenomena by the air ventilation, such as air cavity and water splash due to the waterexit and re-entry of the propeller blade. The air ventilation increases with increasing propeller rotating speed and decreasing submergence depth.

When the propeller rotating speed is at a lower propeller rotating speed, such as 2 rps, the maximum thrust and torque of a blade during a single revolution are larger than the average values of the deep water condition. The change in pressure around the air cavity generated by the tip vortex increases the thrust and torque under the free surface.

The average thrust and torque losses during a single revolution increase with increasing air ventilation. In addition, the thrust loss is larger than the torque loss in all cases with decreasing propeller efficiency. The propeller efficiency decreases with increasing propeller rotating speed and decreasing submergence depth.

The thrust and torque losses were compared with the experimental data and empirical formula reported by Park et al. [1], showing good agreement at different submergence depths and ventilation numbers. Nevertheless, further study will be needed to improve the accuracy of the numerical simulations using the turbulence model such as detached eddy simulation (DES) or large eddy simulation (LES) and extend the empirical formula to various propeller loading conditions and submergence depths. 


\section{ACKNOWLEDGEMENTS}

This work was supported by INHA UNIVERSITY Research Grant (INHA-55428) and Industrial Core Technology Development Program "Technology Development to Improve Added Resistance and Ship Operational Efficiency for Hull Form Design” (10062881) of Ministry of Trade, Industry and Energy, Republic of Korea.

\section{REFERENCES}

[1] Park, H.-G., Lee, T.-G., Paik, K.-J., and Choi, S.-H., "Study on the Characteristics of Thrust and Torque for Partially Submerged Propeller," Journal of the Korean Society for Marine Environmental Engineering, vol. 14, pp. 264-272, 2011. https://doi.org/10.7846/JKOSMEE.2011.14.4.264.

[2] Koushan, K., Spence, S., and Hamstad, T., "Experimental Investigation of the Effect of Waves and Ventilation on Thruster Loadings," First International Symposium on Marine Propulors, Trondheim, Norway, 2009.

[3] Young, Y. L. and Kinnas, S. A., "Performance Prediction of Surface-Piercing Propellers," Journal of Ship Research, vol. 28, no. 4, pp. 288-304, 2004.

[4] Califano, A. and Steen, S., "Numerical simulations of a fully submerged propeller subject to ventilation," Ocean Engineering, vol. 38, pp. 1582-1599, 2011. https://doi.org/10.1016/j.oceaneng.2011.07.010.

[5] Kozlowska, A. M., Steen, S., and Koushan, K., "Classification of Different Type of Propeller Ventilation and Ventilation Inception Mechanism," First International Symposium on Marine Propulors, Trondheim, Norway, 2009.

[6] Kempf, G., "The influence of viscosity on thrust and torque of a propeller working near the surface," Transactions of the Institution of Naval Architects, pp. 321-326, London, UK, 1934.

[7] Shiba, H., "Air-Drawing of Marine Propellers," Transportation Technical Research Institute, Report no. 9, Japan, 1953.

[8] Gutsche, F., "Einfluss der Tauchung auf Schub und Wirkungsgrad von Schiffspropellern," Shiffbauforschung 65/6/67, pp. 256-277, Berlin, Germany, 1967.

[9] Fleischer, K. P., "Untersuchungen über das Zusammenwirken von Schiff und Propeller bei teilgetauchten Propellern," Publication 35/73 of Forschungszentrum des Deutschen Schifbaus, Hamburg, Germany, 1973.

[10] Faltinsen, O., Minsaas, K., Liapias, N., and Skjørdal, S. O., "Prediction of resistance and propulsion of a ship in a seaway," Proceedings of 13th Symposium on Naval Hydrodynamics (Edited by T. Inui), 1981.

[11] Minsaas, K., Faltinsen O., and Person, B., "On the Importance of Added Resistance, Propeller Immersion and Propeller Ventilation for Large Ships in a Seaway," Proceedings of 2nd International Symposium on Practical Design in Shipbuilding (PRADS), Tokyo and Seoul, pp.149-159, 1983.

[12] Furuya, O, "A performance-prediction theory for partially submerged ventilated propellers," Journal of Fluid Mechanics, vol. 151, pp. 311-335, 1985. https://doi.org/10.1017/S0022112085000982.

[13] Koushan, K., "Dynamics of Propeller Blade and Duct Loading on Ventilated Thrusters in Dynamic Positioning Mode," Dynamic Positioning Conference, Houston, USA, 2007. https://doi.org/10.1016/j.oceaneng.2011.07.009.

[14] Califano, A. and Steen, S., "Identification of ventilation regimes of a marine propeller by means of dynamic-loads analysis,” Ocean Engineering, vol. 38, pp. 1600-1610, 2011.

[15] Kozlowska, A. M., Wöckner, K., Steen, S., Rung, T., Kousan, K., and Spence, S. J.B., "Numerical and Experimental Study of Propeller Ventilation," Second International Symposium on Marine Propulors, Hamburg, Germany, 2011.

$\begin{array}{lll}\text { Submitted: } & \text { 08.04.2017. } & \begin{array}{l}\text { Sungwook Lee, swlee@ } @ \text { kmou.ac.kr } \\ \text { Division of Naval Architecture and Ocean Systems } \\ \text { Accepted: }\end{array} \quad \text { 14.11.2017. } \\ & & \begin{array}{l}\text { Korea Maritime and Ocean University, Busan 49112, Republic of Korea } \\ \text { Kwang-Jun Paik, corresponding author, kwangjun.paik@ @inha.ac.kr } \\ \text { Department of Naval Architecture and Ocean Engineering, } \\ \text { Inha University, Incheon 22212, Republic of Korea }\end{array}\end{array}$

